Hurwitz' definition of commutative group employs two postulates; ${ }^{*}$ the reader will find it interesting to compare with that of the present paper.

University of California at Los Angeles

\title{
ON SOME QUADRATURE FORMULAS AND ON ALLIED THEOREMS ON TRIGONOMETRIC POLYNOMIALS
}

\section{BY J. GERONIMUS}

1. Introduction. We consider the following problem.

Find $2 n$ numbers $0 \leqq \theta_{1}<\theta_{2}<\cdots<\theta_{2 n-1}<\theta_{2 n}<2 \pi$ such that for every trigonometric polynomial

(1) $\quad G(\theta)=\alpha_{n}+\sum_{k=0}^{n-1}\left\{\alpha_{k} \cos (n-k) \theta+\beta_{k} \sin (n-k) \theta\right\}$

of order $\leqq n$ the equality

$$
\int_{0}^{2 \pi} F(\theta) G(\theta) d \theta=L\left\{\sum_{i=1}^{n} G\left(\theta_{2 i-1}\right)-\sum_{i=1}^{n} G\left(\theta_{2 i}\right)\right\}
$$

holds true, where $F(\theta)$ is the given function

$$
F(\theta)=\sum_{k=n-s}^{\infty}\left(A_{k} \cos k \theta+B_{k} \sin k \theta\right), \quad(s \leqq(n-1) / 2),
$$

and $L$ is a given positive number.

Let

$$
\begin{aligned}
& F_{n}(\theta)=\sum_{k=n-s}^{n}\left\{A_{k} \cos k \theta+B_{k} \sin k \theta\right\} \\
& p_{n}(\theta)=-\int F_{n}(\theta) d \theta, \quad G^{*}(\theta)=\text { const. } \prod_{k=1}^{2 n} \sin \frac{\theta-\theta_{k}}{2} .
\end{aligned}
$$

Then, integrating (2), we get

\footnotetext{
* For references, see the first footnote to this paper.
} 


$$
\int_{0}^{2 \pi}\left\{p_{n}(\theta)-\frac{L}{2} \operatorname{sgn} G^{*}(\theta)\right\} G^{\prime}(\theta) d \theta=0,
$$

whence it follows that

(5) $\frac{L}{2} \operatorname{sgn} G^{*}(\theta)=\frac{c}{2}+p_{n}(\theta)+\sum_{k=n+1}^{\infty}\left(M_{k} \cos k \theta+N_{k} \sin k \theta\right)$, where $c$ is an arbitrary constant; it is clear that

$$
|c| \leqq L \text {. }
$$

By the theorem of N. Achyeser and M. Krein $†$ a necessary and sufficient condition for the existence of a function deviating from zero by not more than $L / 2$ and having the first members of the Fourier expansion

$$
\frac{c}{2}+R \sum_{k=1}^{n} \bar{c}_{k} z^{k}, \quad c_{k}=a_{k}+i b_{k}, \quad\left(k=1,2, \cdots, n ; z=e^{i \theta}\right)
$$

is that the form

$$
\sum_{r=0}^{n} \sum_{k=0}^{n} \gamma_{r-k} x_{r} \bar{x}_{k}
$$

be non-negative, where the $\gamma$ 's are to be found as the coefficients of the expansion

$$
e^{(\pi i c) /(2 L)} \cdot e^{(\pi i / L) S}=\gamma+\gamma_{1} z+\cdots+\gamma_{n} z^{n}+\cdots
$$

where

$$
S=\sum_{k=1}^{n} \bar{c}_{k} z^{k}
$$

and where $\gamma_{0}=\gamma+\bar{\gamma}, \gamma_{-k}=\bar{\gamma}_{k},(k=1,2, \cdots, n)$.

It is clear that in our case we have

$$
\begin{gathered}
c_{n-k}=\frac{B_{n-k}-i A_{n-k}}{n-k}, \quad \gamma_{n-k}=\frac{\pi i}{L} e^{(\pi i c) /(2 L)} \bar{c}_{n-k}, \\
(k=0,1, \cdots \\
\gamma_{0}=2 \cos \frac{\pi c}{2 L}, \quad \gamma_{1}=\gamma_{2}=\cdots=\gamma_{n-s-1}=0 .
\end{gathered}
$$

$\dagger$ N. Achyeser and M. Krein, Über Fouriersche Reihen beschränkter summierbarer Funktionen und ein neuer Extremumproblem (1 Teil), Transactions of the Kharkow Mathematical Society, vol. 9 (1934), pp. 18-19. 
Setting

$$
i e^{(\pi i c) /(2 L)} x_{n-k}=y_{n-k}, \quad x_{k}=y_{k}, \quad(k=0,1, \cdots, s),
$$

we may write our condition thus:

$$
\frac{2 L}{\pi} \cos \frac{\pi c}{2 L}+A \geqq 0,
$$

where we have put

$$
A=\frac{\sum_{r, k} c_{r-k} y_{k} \bar{y}_{r}}{\sum_{k=0}^{n}\left|y_{k}\right|^{2}}, \quad(n-s \leqq|r-k| \leqq n) .
$$

It is easy to find that $A$ satisfies the inequality

$$
-\delta_{0} \leqq A \leqq \delta_{0},
$$

where $\delta_{0}$ is the greatest root of the equation

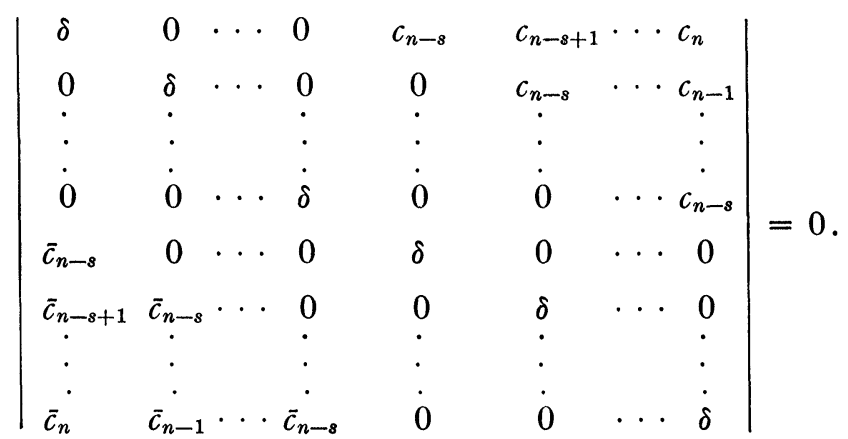

Therefore our form (7) will be non-negative if and only if

$$
\cos \frac{\pi c}{2 L}=\frac{\pi \delta_{0}}{2 L}, \quad L \geqq \frac{\pi \delta_{0}}{2} .
$$

Such are the necessary conditions for the existence of the quadrature formula (2); it is not difficult to see that they are sufficient too.

2. Roots of Trigonometric Polynomials. Thus we are to find the polynomial $G^{*}(\theta)$ of order $\leqq n$ from the condition 


$$
\operatorname{sgn} G^{*}(\theta)=\frac{c}{L}+\frac{2}{L} R \sum_{k=0}^{s} \bar{c}_{n-k} z^{n-k}+R \sum_{k=n+1}^{\infty} \mu_{k} z^{k}, \quad\left(z=e^{i \theta}\right) .
$$

Consider the polynomial

$$
P(\theta, \alpha)=R\left[q^{2}(z) z^{n-2 s+\nu}\right]+|q(z)|^{2} \cos \alpha, \quad\left(z=e^{i \theta}\right),
$$

where $\nu+1$ is the multiplicity of $\delta_{0}$ and the polynomial $q(z)$ of degree $s-\nu$ is to be found from the condition

$$
\begin{aligned}
\delta_{0} \frac{q(z)}{z^{s-\nu} \bar{q}(1 / z)}=\bar{c}_{n-s}+\bar{c}_{n-s+1} z+\cdots+\bar{c}_{n} z^{s}+\cdots, & (|z| \leqq 1) .
\end{aligned}
$$

We have

sgn $P(\theta, \alpha)$

$$
=\operatorname{sgn}\left\{\cos \frac{(n-2 s+\nu) \theta+2 \vartheta+\alpha}{2} \cos \frac{(n-2 s+\nu) \theta+2 \vartheta-\alpha}{2}\right\},
$$

where $\vartheta=\arg q(z)$, whence we find

$$
\begin{aligned}
& \operatorname{sgn} P(\theta, \alpha)=\operatorname{sgn}\left\{e^{-i \alpha} \frac{1+z^{n-s} e^{i \alpha} \frac{q(z)}{z^{s-v} \bar{q}(1 / z)}}{1+z^{n-s} e^{-i \alpha} \frac{q(z)}{z^{s-v} \bar{q}(1 / z)}}\right\} \\
& =R\left\{1-\frac{2 \alpha}{\pi}+\frac{2}{\pi i} \log \frac{1+z^{n-s} e^{i \alpha} \frac{q(z)}{z^{s-\nu} \bar{q}(1 / z)}}{1+z^{n-s} e^{-i \alpha} \frac{q(z)}{z^{s-\nu} \bar{q}(1 / z)}}\right\} \\
& =1-\frac{2 \alpha}{\pi}+\frac{4 \sin \alpha}{\pi \delta_{0}} R \sum_{k=0}^{s} \bar{c}_{n-k} z^{n-k}+R \sum_{k=n+1}^{\infty} \lambda_{k} z^{k} \text {. }
\end{aligned}
$$

On putting

$$
1-\frac{2 \alpha}{\pi}=\frac{c}{L}, \quad(0 \leqq \alpha \leqq \pi)
$$

we see that

$$
\operatorname{sgn} G^{*}(\theta)-\operatorname{sgn} P(\theta, \alpha)=\sum_{k=n+1}^{\infty}\left(M_{k} \cos k \theta+N_{k} \sin k \theta\right) .
$$


Hence it follows that almost everywhere in the interval $(0,2 \pi)$

$$
\operatorname{sgn} G^{*}(\theta)=\operatorname{sgn} P(\theta, \alpha),
$$

for we have

$$
\int_{0}^{2 \pi}\left[\operatorname{sgn} G^{*}(\theta)-\operatorname{sgn} P(\theta, \alpha)\right] G^{*}(\theta) d \theta=0,
$$

and the integrand is non-negative. Hence we have the following theorem.

ThEOREM 1. Being given the function

$$
F(\theta)=\sum_{k=n-s}^{\infty}\left\{A_{k} \cos k \theta+B_{k} \sin k \theta\right\}, \quad(s \leqq(n-1) / 2),
$$

and the positive number $L$, we can find $2 m \leqq 2 n$ real numbers $0 \leqq \theta_{1}<\theta_{2}<\cdots<\theta_{2 m}<2 \pi$ such that the quadrature formula

$$
\int_{0}^{2 \pi} F(\theta) G(\theta) d \theta=L\left\{\sum_{i=1}^{m} G\left(\theta_{2 i-1}\right)-\sum_{i=1}^{m} G\left(\theta_{2 i}\right)\right\}
$$

holds true for every trigonometric polynomial $G(\theta)$ of order $\leqq n$; $L$ must satisfy the inequality $L \geqq \pi \delta_{0} / 2$, where $\delta_{0}$ is the greatest root of (13); $c_{n-k}=\left(B_{n-k}-i A_{n-k}\right) /(n-k), \quad(k=0,1, \cdots, s)$; $m=n-\nu$, where $\nu+1$ is the multiplicity of $\delta_{0}$; the numbers $\theta_{1}, \theta_{2}, \cdots, \theta_{2 m}$ are the roots of the trigonometric polynomial of order $m$

$$
\begin{aligned}
G^{*}(\theta) & =P(\theta, \alpha) \\
& =R\left[q^{2}(z) z^{n-2 s+\nu}\right] \pm|q(z)|^{2}\left(1-\left(\frac{\pi \delta_{0}}{2 L}\right)^{2}\right)^{1 / 2},
\end{aligned}
$$

where the polynomial $q(z)$ of degree $s-\nu$ is to be found from the expansion (16).

3. Minima of Polynomials. Putting

$$
1-\frac{2 \alpha}{\pi}=\lambda, \quad(-1 \leqq \lambda \leqq 1),
$$

we obtain the equality

$$
\int_{0}^{2 \pi} \operatorname{sgn} G^{*}(\theta) G(\theta) d \theta=\lambda \int_{0}^{2 \pi} G(\theta) d \theta+\frac{4}{\delta_{0}} \cos \frac{\pi \lambda}{2}
$$


which is valid for every trigonometric polynomial

$$
G(\theta)=\alpha_{n}+\sum_{k=0}^{n-1}\left\{\alpha_{k} \cos (n-k) \theta+\beta_{k} \sin (n-k) \theta\right\}
$$

satisfying the condition

$$
\omega(G)=\sum_{k=0}^{s}\left(\alpha_{k} a_{n-k}+\beta_{k} b_{n-k}\right)=1, \quad(s \leqq(n-1) / 2) .
$$

Hence follows the inequality

$$
\int_{0}^{2 \pi}|G(\theta)| d \theta-\lambda \int_{0}^{2 \pi} G(\theta) d \theta \geqq \frac{4}{\delta_{0}} \cos \frac{\pi \lambda}{2},
$$

where the equality holds true only for

$$
G(\theta)=G^{*}(\theta)=P(\theta, \alpha), \quad(\lambda=1-2 \alpha / \pi) .
$$

First put $\lambda=0$; then we get

$$
\int_{0}^{2 \pi}|G(\theta)| d \theta \geqq \frac{4}{\delta_{0}}
$$

Suppose now that $G(\theta)$ is non-negative; then

$$
\int_{0}^{2 \pi} G(\theta) d \theta \geqq \frac{4}{\delta_{0}} \frac{\cos (\pi \lambda / 2)}{1-\lambda} .
$$

Supposing that $\lambda \rightarrow 1$, we get finally

$$
\int_{0}^{2 \pi} G(\theta) d \theta \geqq \frac{2 \pi}{\delta_{0}}
$$

the equality is valid for the polynomial

$$
G(\theta)=P(\theta, 0)=|q(z)|^{2}+R\left[q^{2}(z) z^{n-2 s+\nu}\right] .
$$

We have thus proved the following theorem.

THEOREM 2. For every trigonometric polynomial

$$
G(\theta)=\alpha_{n}+\sum_{k=0}^{n-1}\left\{\alpha_{k} \cos (n-k) \theta+\beta_{k} \sin (n-k) \theta\right\}
$$

satisfying the condition 


$$
\omega(G)=\sum_{k=0}^{s}\left\{\alpha_{k} a_{n-k}+\beta_{k} b_{n-k}\right\}=1, \quad(s \leqq(n-1) / 2),
$$

the inequality

$$
\int_{0}^{2 \pi}|G(\theta)| d \theta \geqq \frac{4}{\delta_{0}}
$$

is valid; the polynomial for which this minimum is actually attained is

$$
P\left(\theta, \frac{\pi}{2}\right)=R\left[q^{2}(z) z^{n-2 s+\nu}\right] .
$$

If the polynomial is bound to be non-negative, then the minimum will be $\pi / 2$ times (26) and this minimum will be attained by the polynomial

$$
P(\theta, 0)=|q(z)|^{2}+R\left[q^{2}(z) z^{n-2 s+\nu}\right],
$$

where $q(z), \delta_{0}$, and $\nu$ have the same meaning as in Theorem 1.

MatheMatical InstituTE,

KHARKOW, U.S.S.R. 